\title{
ICRH system performance during ITER-Like Wall operations at JET and the outlook for DT campaign
}

\author{
Igor Monakhov ${ }^{1, *}$, Trevor Blackman ${ }^{1}$, Pierre Dumortier ${ }^{1,2}$, Frederic Durodié ${ }^{2}$, Philippe Jacquet ${ }^{1}$, Ernesto Lerche Er, $^{1,2}$ \\ Craig Noble ${ }^{1}$, and JET Contributors ${ }^{3}$ \\ ${ }^{1}$ CCFE, Culham Science Centre, Abingdon, OX14 3DB, United Kingdom \\ ${ }^{2} \mathrm{LPP}-\mathrm{ERM} / \mathrm{KMS}$, TEC partner, Brussels, Belgium \\ ${ }^{3}$ See the author list of "Overview of the JET results in support to ITER" by X. Litaudon et al. to be published in Nuclear Fusion \\ Special issue: overview and summary reports from the 26th Fusion Energy Conference (Kyoto, Japan, 17-22 October 2016)
}

\begin{abstract}
Performance of JET ICRH system since installation of the metal ITER-Like Wall (ILW) has been assessed statistically. The data demonstrate steady increase of the RF power coupled to plasmas over recent years with the maximum pulse-average and peak values exceeding respectively $6 \mathrm{MW}$ and $8 \mathrm{MW}$ in 2016. Analysis and extrapolation of power capabilities of conventional JET ICRH antennas is provided and key performance-limiting factors are discussed. The RF plant operational frequency options are presented highlighting the issues of efficient ICRH application within a foreseeable range of DT plasma scenarios.
\end{abstract}

\section{Introduction}

In 2011, the carbon first wall at JET was replaced with a metal ITER-Like Wall (ILW). Reliable injection of high RF power into ELMy plasmas is essential for control of heavy impurities in high-performance JET discharge scenarios [1]. This requirement fully applies to planned DT experiments which, in addition, will benefit from high-power ICRH for boosting the fusion reaction [2].

\section{Status of the RF plant at JET}

The RF plant at JET (Fig. 1) comprises four conventional 4-strap antennas energised in pairs by two different power-splitting systems [3,4] and one 8-strap main-port ITER-Like Antenna (ILA) [5]. The ELM-tolerant $3 \mathrm{~dB}$ hybrid system (antennas $\mathrm{A} \& \mathrm{~B}$ ) is operational since 2006. The External Conjugate-T (ECT) system (antennas C\&D) was installed in 2009 to provide ELM-tolerant operations at four selected frequencies [4]; in 2016, the number of commissioned ECT frequencies was increased to eleven covering the $25-57 \mathrm{MHz}$ band. It has to be noted that the existing ECT transmission line configuration does not allow achieving the ELM-tolerance at frequencies close to $25 \mathrm{MHz}, 29 \mathrm{MHz}$ and 37MHz. The ILA was first introduced in 2008; however it remained inactive during initial period of ILW operations due to a capacitor failure. In 2015, the system was restored and successfully recommissioned at several frequencies within the $29-51 \mathrm{MHz}$ band demonstrating adequate tolerance to moderate ELMs. Presently, one half of the ILA is under investigation for emerged capacitor control problems which may affect its availability in future.

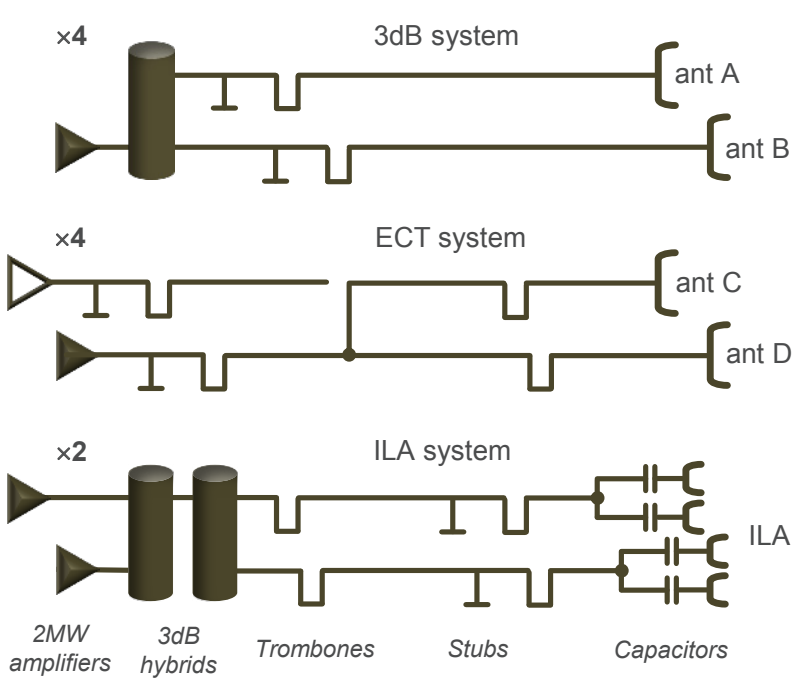

Fig. 1. Schematic representation of three independent RF systems used during ILW ICRH operations at JET.

\section{Total RF power coupled to ILW plasmas}

Statistical data (Fig. 2) demonstrate the steady increase of maximum and average total RF power coupled to plasma during JET pulses since the ILW installation. This encouraging trend can be attributed to several factors including the re-introduction of the ILA in 2015, development of gas injection scenarios for coupling improvement [6], achieving statistically higher maximum antenna voltages (see Sect. 3.1), growing operator experience and ongoing efforts to improve diagnostics, tuning and maintenance of the RF plant subsystems. In 2016, the peak and pulse-average total

\footnotetext{
* Corresponding author: igor.monakhov@,ukaea.uk
} 
coupled power reached respectively $8 \mathrm{MW}$ and $6 \mathrm{MW}$; notably, comparable results were achieved in low-, medium- and high-frequency bands. Due to poor coupling, high-power operations at low frequencies remain challenging for the conventional JET antennas (see Sect. 3.2) and availability of the ILA is essential for delivering high $\mathrm{RF}$ power to plasmas at $\mathrm{f}<35 \mathrm{MHz}$.

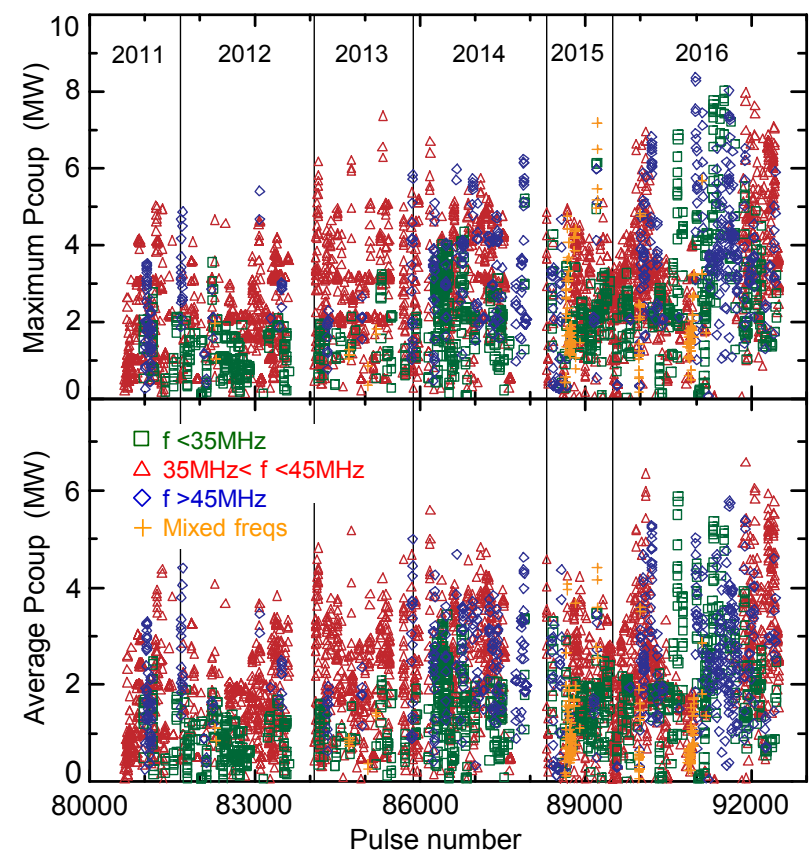

Fig. 2. The maximum (top) and average (bottom) total RF power coupled to plasma during JET ILW pulses in different frequency bands.

\section{Capabilities of conventional antennas}

\subsection{Statistics for performance-limiting factors}

Technically, power limitations of conventional ICRF antennas at JET can be attributed to two principal factors: antenna \& transmission line arcing at high voltages and performance issues and fault-free power capabilities of generators feeding the antenna straps.

Figure 3 provides statistics for the maximum voltages $V_{\text {strap }}^{\max }$ reached on the antenna straps during JET pulses. The data indicates that higher voltages have been routinely achieved since the ILW installation. Although open to other interpretations, the increase can be related to improved electrical strength of the RF system due to ongoing transmission line refurbishments and less dusty carbon-free ILW vacuum environment. The operational $V_{\text {strap }}^{\max }$ limit of $30-35 \mathrm{kV}$ is rather fuzzy and it is subject not only to variable physical thresholds of arcing but also to a number of practical compromises (conditioning/deconditioning, probability/confidence, risk/gain etc.).

Statistical assessment of the RF generator performance is given on Fig. 4. It clearly shows that even in presence of the power-splitting $3 \mathrm{~dB} \& \mathrm{ECT}$ systems, the $2 \mathrm{MW}$ generators typically remain loaded only partially, i.e. hardware upgrades for higher nominal maximum power output are not needed. At the same time, improving reliability and fault-free performance of the existing generators, especially at the output power above $1 \mathrm{MW}$, is necessary for enhancing coupled power capabilities of the conventional antennas. In preparation for the DT campaign, a modern FPGA-based data collection system is being installed at JET which will allow in-depth diagnostics of the RF amplifier stages and facilitate their tuning, optimisation and fault-finding.

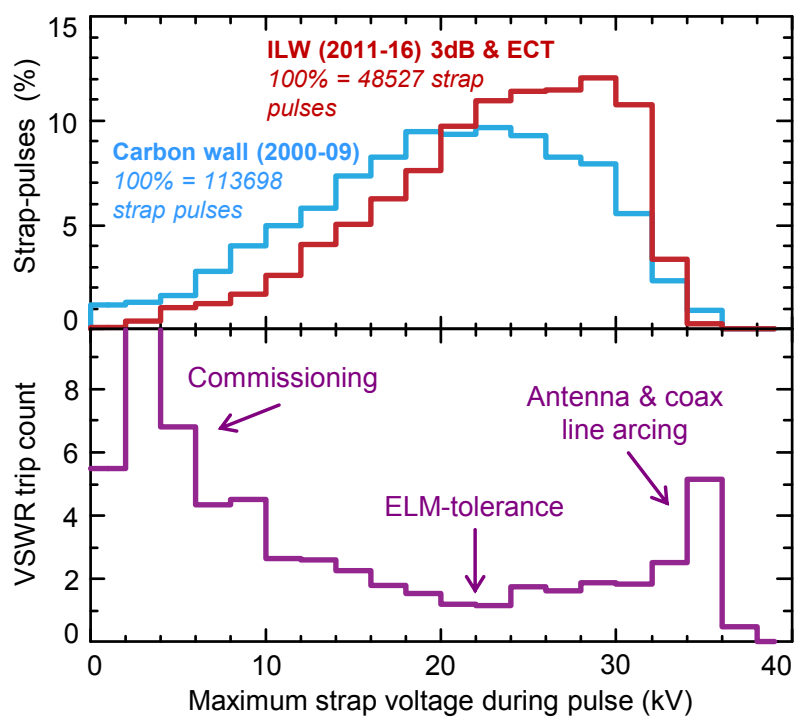

Fig. 3. Percentage of antenna strap pulses reaching given maximum voltage during the carbon-wall and ILW operations (top) and the average number of high-VSWR protection trips during a ILW pulse per the RF generator feeding the strap at given maximum voltage (bottom).

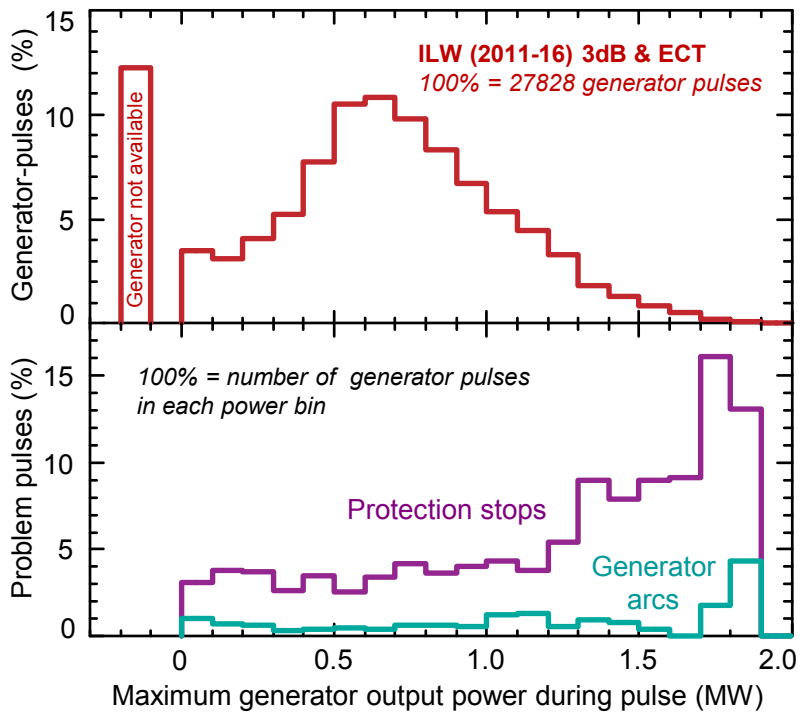

Fig. 4. Percentage of generator pulses reaching given output power during the ILW operations (top) and percentage of problematic generator pulses in each power bracket (bottom).

\subsection{Actual \& feasible maximum coupled power}

The power characteristics of conventional ICRH antennas energised by the $3 \mathrm{~dB} \&$ ECT systems during the ILW pulses are illustrated by Fig. 5. An assessment of feasible maximum power during same pulses is also given in assumption of perfect RF plant control and 
performance within realistic hardware limits. Note that, in practice, the maximum voltages $V_{\text {strap }}^{\max }$ and coupling resistances $R_{\text {strap }}$ of individual antenna straps are different and it is more convenient to use global parameters of strap-equalised maximum voltage $V_{e q}^{\max }$ and strap-averaged coupling resistance $R_{\text {aver }}$ such that:

$$
\begin{aligned}
& P_{\text {coup }}=\sum P_{\text {strap }}=\frac{1}{2 Z_{0}^{2}} \sum\left(V_{\text {strap }}^{\text {max }}\right)^{2} R_{\text {strap }} \equiv \\
& \equiv \frac{\left(V_{e q}^{\text {max }}\right)^{2}}{2 Z_{0}^{2}} \sum R_{\text {strap }}=\frac{n\left(V_{e q}^{\text {max }}\right)^{2}}{2 Z_{0}^{2}} R_{\text {aver }}
\end{aligned}
$$

where $n=16$ is the total number of straps in the conventional antennas and $Z_{0}=30 \mathrm{Ohm}$ is the characteristic impedance of transmission lines at JET.

Figure 5 demonstrates that both the actual $(<7 \mathrm{MW})$ and feasible (4-10MW) maximum power coupled by the antennas strongly depends on specific discharge conditions which define an order of magnitude spread of $R_{\text {aver }}$ during the ILW operations. For this reason, giving universal predictions of antenna power capabilities for the DT campaign is extremely challenging and optimisation of discharge scenarios with respect to antenna-plasma coupling will remain as important as the RF plant optimisation.

The nature of antenna power limitations largely depends on operational frequency: at $\mathrm{f}<35 \mathrm{MHz}$, the coupling resistance is statistically noticeably lower than in the mid- and high-frequency bands. Thus, at low frequencies, the maximum voltage constraints typically dominate over generator performance issues. Antenna coupling and power capabilities in the mid- and highfrequency bands are similar while the generator issues play more significant role than the $V_{\text {strap }}^{\max }$ limitations.
The gap between data points representing the actual and feasible maximum power during the ILW pulses (Fig. 5) indicates that the conventional ICRH antennas at JET have not achieved their full potential yet. Together with hardware performance and reliability improvements, intelligent real-time power balance control between the individual antenna straps and generators is required for bridging this gap. The necessity for such a control can be explained as follows: the limits of fault-free operations and protection thresholds are individual to the 16 antenna straps and 8 generators; the existing real-time power control software responds to changing plasma conditions by modifying the generator global output power request, however it is not capable of automatically adjusting the power balance between the generators during a pulse such that the straps voltages remain optimally distributed within the antennas while the generators stay within their individual operational limits. Consequently, attempts of the ICRH performance maximisation often result in the power being clamped by protection systems or, worse, with the RF pulse abnormally terminated due to issues with a single weakest component while the rest of the RF plant has not reached its limits. Inadequate balance optimisation also explains the significant difference between the maximum voltages achieved on individual antenna straps (Fig. 3) and the strap-equalised maximum voltages (Fig. 5) characterising the plant as a whole. The existing situation requires from the RF plant operators to make several optimisation pulses and to leave substantial fail-safe confidence margins while reaching the maximum coupled power. A major upgrade of real-time RF power control software is presently underway at JET which will address the aforementioned issues.
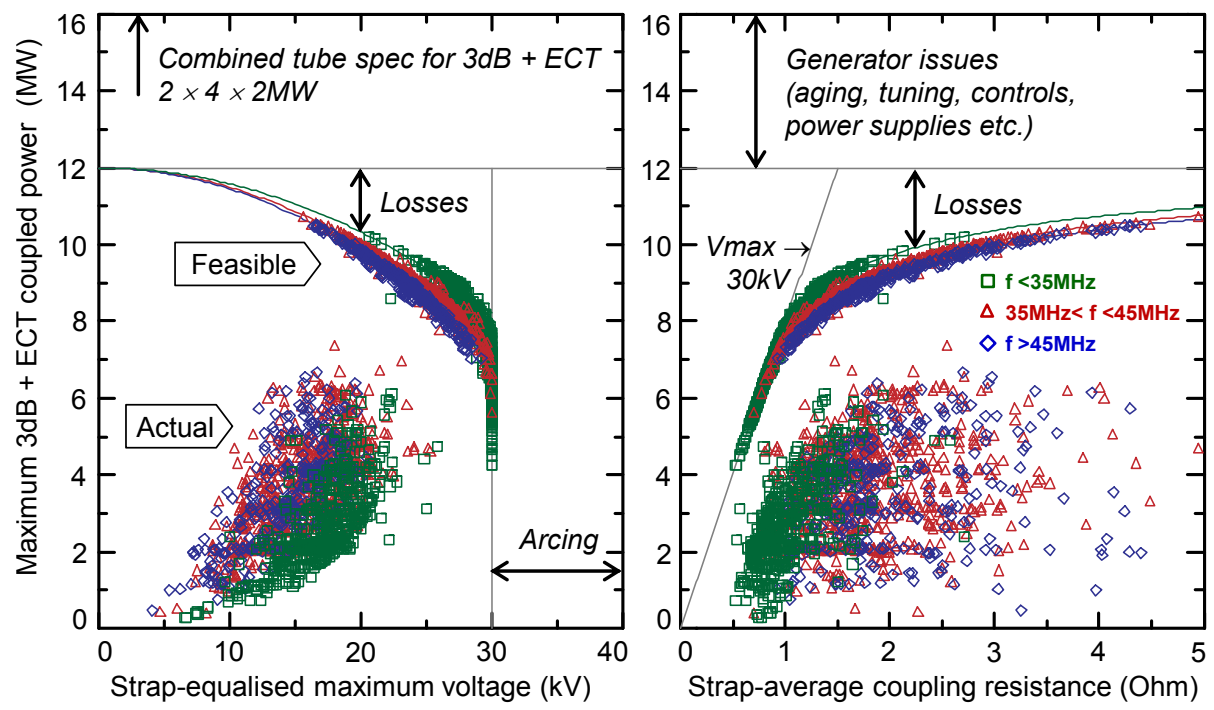

Fig. 5. Dependencies of actual and feasible maximum RF powers coupled to the ILW plasmas during combined operations of the $3 \mathrm{~dB}$ and ECT systems on the strap-equalised maximum voltage (left) and on the strap-average coupling resistance (right). Each symbol represents a JET pulse with all the 16 straps of conventional antennas operational; the values of the maximum voltage and coupling resistance correspond to the moment of time during a pulse when the total power coupled by the $3 \mathrm{~dB} \& \mathrm{ECT}$ systems reaches the maximum. Feasible power is assessed on the basis of coupling resistances of individual straps measured during the actual pulses and assuming perfect RF plant performance within $30 \mathrm{kV}$ maximum strap voltage limit and $75 \%$ power capability of each generator. 


\section{Operational frequency options and implications for DT ICRH scenarios}

Efficient ICRH application in a range of possible DT scenarios with central toroidal magnetic field within $\mathrm{B}_{\mathrm{T}}=2.7-4.0 \mathrm{~T}$ requires careful consideration of the $\mathrm{RF}$ plant operational frequency options. The frequencies and corresponding coupled power capabilities of individual RF systems are subject to a number of constraints related to transmission line configuration, matching element variation and antenna RF characteristics. The ICRH-assisted tungsten control and enhancement of DT fusion reaction rely on different ICRH mechanisms which may require operations at different frequencies. Additional difficulties arise from undesirable presence of ion cyclotron resonances on the inner or outer vessel walls at certain frequencies; such potentially harmful conditions are avoided thanks to operational instructions presently adopted at JET.

Figure 6 summarises the available frequency options for the $3 \mathrm{~dB}$, ECT and ILA systems and shows corresponding $\mathrm{B}_{\mathrm{T}}$ compatibility windows for the $\mathrm{RF}$ power deposition in central plasma regions (specifically, in the regions where relevant ion cyclotron resonances are within $-5 \mathrm{~cm}<\mathrm{R}-\mathrm{R}_{0}<+15 \mathrm{~cm}$ ). Note that the system maximum powers refer to all kind of ILW plasmas in 2011-16, i.e. the data are not directly relevant to ELMy high-performance DT discharges. At the same time, no changes to the commissioned frequencies of the $3 \mathrm{~dB} \& \mathrm{ECT}$ systems are expected unless the transmission and matching hardware is modified.

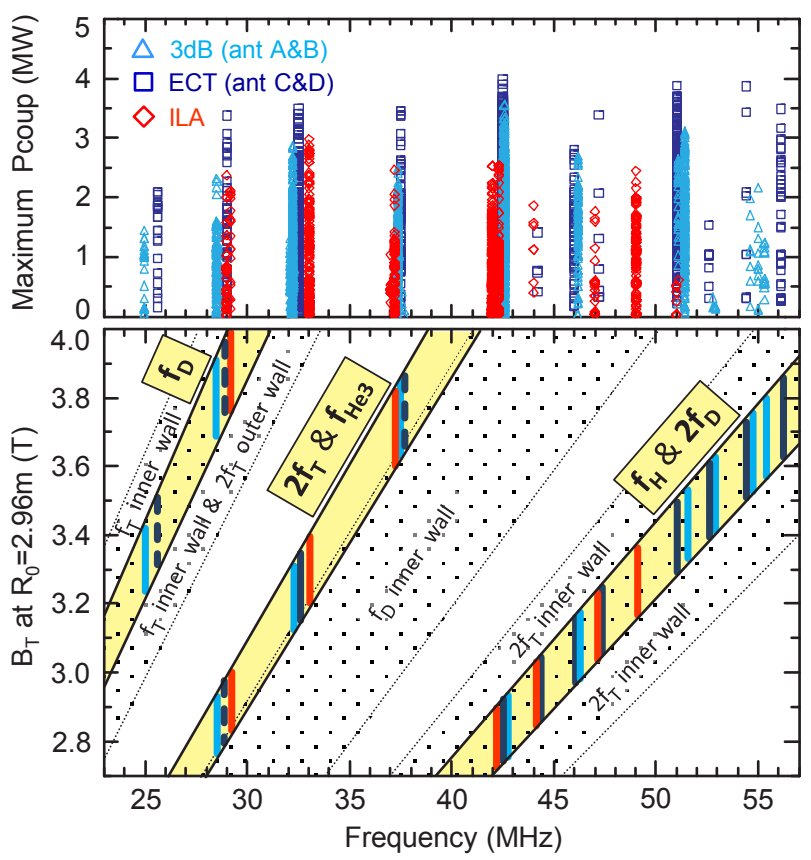

Fig. 6. Maximum power coupled to ILW plasmas by individual RF systems at the commissioned frequencies (top) and toroidal magnetic field induction at the JET vessel centre required for efficient ICRH application during DT experiments depending on the operational frequency (bottom). Shaded areas indicate undesirable conditions when parasitic ion cyclotron resonances will be present on the inner or outer vessel walls.
On the basis of currently available information, the outlook for applicability of main ICRH scenarios during the DT experiments at JET is the following:

- $\mathrm{f}=\mathrm{f}_{\mathrm{H}}=2 \mathrm{f}_{\mathrm{D}}-$ good coverage of the $\mathrm{B}_{\mathrm{T}}$ range; high power expectations regardless of the ILA availability; unavoidable inner wall $\mathrm{f}=2 \mathrm{f}_{\mathrm{T}}$ resonance

- $\quad \mathrm{f}=2 \mathrm{f}_{\mathrm{T}}=\mathrm{f}_{\mathrm{He}}$ - reduced coverage of the $\mathrm{B}_{\mathrm{T}}$ range; high power operations require the ILA; no major issues with the wall resonances

- $\mathrm{f}=\mathrm{f}_{\mathrm{D}}-$ low power expectations except for the ILAassisted high- $\mathrm{B}_{\mathrm{T}}$ option; unavoidable inner wall $\mathrm{f}=\mathrm{f}_{\mathrm{T}}$ and marginally avoidable outer wall $\mathrm{f}=2 \mathrm{f}_{\mathrm{T}}$ resonances Combining the ICRH heating schemes by simultaneously operating different RF systems in different frequency bands might be advantageous both for maximising the total RF coupled power and the DT physics program. Dedicated assessment of implications of the vessel wall $\mathrm{f}=\mathrm{f}_{\mathrm{T}} \& \mathrm{f}=2 \mathrm{f}_{\mathrm{T}}$ parasitic resonances in presence of tritium neutral beam injection needs to be done in preparation to the DT campaign.

\section{Conclusions}

Steady progress has been made at JET in increasing the total RF power coupled to plasma since installation of ILW; the maximum pulse-average and peak values reached respectively $6 \mathrm{MW}$ and $8 \mathrm{MW}$ in 2016 . Further efforts are required for improving reliability \& performance of the RF generators and implementation of advanced real-time power balance control which will allow approaching the full potential of the RF plant.

At the same time, the maximum power coupled to plasma at present and the expectations for future DT campaign strongly depend on antenna-plasma coupling conditions specific to particular discharge scenarios and on the $\mathrm{B}_{\mathrm{T}}$-dependent selection of the $\mathrm{RF}$ plant operational frequencies.

The main concerns for high-power ICRH operations during the DT campaign are related to uncertainties of the ILA availability in view of recently emerged capacitor control issues, insufficient ELM-tolerance of the ECT system at $25 \mathrm{MHz}, 29 \mathrm{MHz} \& 37 \mathrm{MHz}$ and the presence of parasitic tritium cyclotron resonances on the vessel inner wall.

This work has been carried out within the framework of the EUROfusion Consortium and has received funding from the Euratom research and training programme 2014-2018 under grant agreement No 633053. The views and opinions expressed herein do not necessarily reflect those of the European Commission.

\section{References}

1. E. Lerche, et al., Nucl. Fusion $56036022(2016)$

2. M. J. Mantsinen, et al., this conference

3. M. Graham, et al., Plasma Phys. Control. Fusion 54, 074011 (2012)

4. I. Monakhov, et al., Nucl. Fusion 53, 083013 (2013)

5. F. Durodié, et al., Plasma Phys. Control. Fusion 54, 074012 (2012)

6. P. Jacquet, et al., Nucl. Fusion 56, 046001 (2016) 